\title{
Stability and Well-posedness of a Nonlinear Railway Track Model
}

\author{
M. Sajjad Edalatzadeh and Kirsten A. Morris
}

\begin{abstract}
Railway tracks rest on a foundation known for exhibiting nonlinear viscoelastic behavior. Railway track deflections are modeled by a semilinear partial differential equation. This paper studies the stability of solutions to this equation in presence of an input. With the aid of a suitable Lyapunov function, existence and exponential stability of classical solutions is established for certain inputs. The Lyapunov function is further used to find an a-priori estimate of the solutions, and also to study the input-to-state stability (ISS) of mild solutions.
\end{abstract}

Index Terms-Distributed parameter systems, flexible structures, partial differential equations, nonlinear systems, input-tostate stability.

\section{INTRODUCTION}

$\mathbf{S}$ TABILITY analysis of nonlinear partial differential equations (PDE's) modeling flexible structures has attracted attention in the past few decades. To name but a few of the publications in this field; in [1], b oundary stabilization of a nonlinear beam clamped at one end and supported by a nonlinear bearing at the other end is studied. In [2], authors investigated asymptotic behavior of a semilinear viscoelastic beam model including a memory term. The nonlinearity is assumed to satisfy some growth assumption. In [3], asymptotic stability of Falk model of shape memory alloys is studied using energy method. In [4], [5], boundary stabilization of a nonlinear micro-beam model is studied using linearizion technique together with Lyapunov method. The von Karman model of slender beams has also been investigated in many papers. In a recent study, Liu et al. considered asymptotic stability of von Karman beam with thermo-viscoelastic damping [6]. Nonlinear PDE's with a fourth order spatial derivative are not limited to flexible s tructures; a n e xample i s CahnHilliard equation with inertial term which describes the phase separation of binary fluids; s ee for example, [7].

The nonlinear railway track model in this paper was described in [8]. The nonlinearity in this model is caused by the railway support ballast which is known for highly nonlinear viscoelastic behavior [9]. Another nonlinear model for railway tracks has been suggested [10]. Unlike the previous model, this model also includes shear deformations in flexible track beams. This railway track model was used to study the effect of passing vehicles on pavements [11]. There are also studies devoted to the vibration monitoring and control of

M. S. Edalatzadeh is with the Department of Applied Mathematics, University of Waterloo, Waterloo, Ontario, Canada msedalat@uwaterloo.ca

K. A. Morris is with Faculty of Mathematics, Applied Mathematics, University of Waterloo, Waterloo, Ontario, Canada kmorris@uwaterloo. ca

(c)2018 IEEE railway tracks [12], [13]. Track deflections induced by train passage are a cause of ride discomfort, fatigue in railway, and disturbances to nearby buildings [12]. Thus, the vibrations need to be carefully monitored and controlled.

This paper focuses on well-posedness and stability, with respect to both initial conditions, and inputs of this model. Inputto-state stability (ISS) does not generalize in a straightforward way to infinite dimensions; see [14] for counter examples. Input-to-state stability theory has been extended in recent years to include systems of infinite dimension. In [15], a comparison between ISS theory of finite-dimensional systems and that of infinite-dimensional systems is presented. In [16], ISS of a class of linear infinite dimensional control systems with nonlinear feedbacks is discussed. The nonlinear feedback satisfies a sector condition that does not apply to the nonlinearity in this paper. In [17], strict Lyapunov functions were used to investigate the stability of nonlinear heat equation; also, such Lyapunov functions were used to establish a robust stability of the equation in presence of a convection term and uncertainties. Integral input-to-state stability is a weaker property than ISS. In [18], integral ISS is discussed. In [19], the relation between iISS and ISS is discussed for linear systems with an unbounded control operator. ISS with respect to boundary inputs and disturbances has also been studied in [20]-[22]. In [22], ISS of the reaction-diffusion-advection equation with boundary and in-domain point-wise sensing and actuation is considered.

In this paper, a Lyapunov function is used to establish ISS for the nonlinear controlled railway track model. To construct the Lyapunov function, the multiplier method [23] is used. Furthermore, a density argument is used to prove the ISS of the model when the inputs are not differentiable. In such cases, the Lyapunov function is also non-differentiable. A density argument was also used in [24, Lemma 2.2.3], where the control system is assumed to have a transition map that continuously depends on both initial conditions and inputs.

The paper is organized as follows: Section 2 is a short section containing notation and definitions. Section 3 introduces the railway track model and discusses well-posedness of this model. Section 4 presents existence and exponential stability of classical solutions to the PDE while section 5 is devoted to the stability of mild solutions.

\section{NOTATION AND DEFINITIONS}

Let $\mathbb{X}$ and $\mathbb{U}$ be Banach spaces, and $I \subset \mathbb{R}$ be a possibly unbounded interval. The function space $C^{m}(I ; \mathbb{X})$ consists of all $m$ times continuously differentiable $\mathbb{X}$-valued functions, 
$L_{l o c}^{2}(I ; \mathbb{U})$ is the space of all strongly measurable functions $\boldsymbol{u}: I \rightarrow \mathbb{U}, t \mapsto \boldsymbol{u}(t)$, for which $\|\boldsymbol{u}(t)\|_{\mathbb{U}}$ is in $L_{l o c}^{2}(I, \mathbb{R})$. Denoted by $P C\left(\mathbb{R}^{+} ; \mathbb{U}\right)$ is the space of all bounded, piecewise continuous $\mathbb{U}$-valued function over $\mathbb{R}^{+}$.

The comparison function sets $\mathscr{K}, \mathscr{K}_{\infty}, \mathscr{L}$, and $\mathscr{K} \mathscr{L}$ are defined as

$$
\begin{aligned}
\mathscr{K}:= & \left\{\gamma: \mathbb{R}^{+} \rightarrow \mathbb{R}^{+} \mid \gamma\right. \text { is continuous, } \\
& \text { strictly increasing, and } \gamma(0)=0\}, \\
\mathscr{K}_{\infty}:= & \{\gamma \in \mathscr{K} \mid \gamma \text { is unbounded }\}, \\
\mathscr{L}:= & \left\{\gamma: \mathbb{R}^{+} \rightarrow \mathbb{R}^{+} \mid \gamma\right. \text { is continuous, } \\
& \text { strictly decreasing, and } \left.\lim _{t \rightarrow \infty} \gamma(t)=0\right\}, \\
\mathscr{K} \mathscr{L}:= & \left\{\beta: \mathbb{R}^{+} \times \mathbb{R}^{+} \rightarrow \mathbb{R}^{+} \mid \beta\right. \text { is continuous, } \\
& \beta(\cdot, t) \in \mathscr{K}, \beta(r, \cdot) \in \mathscr{L}, \forall t \geq 0, \forall r>0\} .
\end{aligned}
$$

Let $\boldsymbol{x}$ indicate the state, and $\boldsymbol{u}$ the input. For linear operators $\mathcal{A}: D(\mathcal{A}) \subset \mathbb{X} \rightarrow \mathbb{X}, \mathcal{B}: \mathbb{U} \rightarrow \mathbb{X}$, and possibly nonlinear operator $\mathcal{F}(\cdot): \mathbb{X} \rightarrow \mathbb{X}$, consider the initial value problem

$$
\left\{\begin{array}{l}
\dot{\boldsymbol{x}}(t)=\mathcal{A} \boldsymbol{x}(t)+\mathcal{F}(\boldsymbol{x}(t))+\mathcal{B} \boldsymbol{u}(t), t>0, \\
\boldsymbol{x}(0)=\boldsymbol{x}_{0} \in \mathbb{X} .
\end{array}\right.
$$

Definition 2.1: [25, Definition 4.2.1] (Classical Solution) A function $\boldsymbol{x}:[0, T) \rightarrow \mathbb{X}$ is a classical solution to (IVP) on $[0, T)$ if $\boldsymbol{x}$ is continuous on $[0, T)$, continuously differentiable on $(0, T), \boldsymbol{x}(t) \in D(\mathcal{A})$ for $0<t<T$, and (IVP) is satisfied.

Definition 2.2: (Mild Solution) Let $\mathcal{A}$ be the infinitesimal generator of a strongly continuous semigroup $\mathcal{T}(t)$. If $\boldsymbol{x} \in$ $C([0, T] ; \mathbb{X})$ satisfies

$$
\begin{aligned}
\boldsymbol{x}(t)= & \mathcal{T}(t) \boldsymbol{x}_{0} \\
& +\int_{0}^{t} \mathcal{T}(t-s) \mathcal{F}(\boldsymbol{x}(s)) d s+\int_{0}^{t} \mathcal{T}(t-s) \mathcal{B} \boldsymbol{u}(s) d s,
\end{aligned}
$$

for every $\boldsymbol{x}_{0} \in \mathbb{X}$, it is said to be a mild solution to (IVP).

In the following definitions, it is assumed that a unique mild solution to (IVP) exists for any $\boldsymbol{u} \in P C\left(\mathbb{R}^{+} ; \mathbb{U}\right)$.

Definition 2.3: [15, Definition 9] (Input-to-state Stability) The (IVP) is called input-to-state stable (ISS) if there exist $\beta \in$ $\mathscr{K} \mathscr{L}$ and $\gamma \in \mathscr{K}$ such that for all $\boldsymbol{x}_{0} \in \mathbb{X}, \boldsymbol{u} \in P C\left(\mathbb{R}^{+} ; \mathbb{U}\right)$, and $t>0$ the mild solution (5) satisfies

$$
\|\boldsymbol{x}(t)\| \leq \beta\left(\left\|\boldsymbol{x}_{0}\right\|, t\right)+\gamma\left(\sup _{t \geq 0}\|\boldsymbol{u}(t)\|_{\mathbb{U}}\right) .
$$

The Dini derivative of a function $V: D(\subset \mathbb{X}) \rightarrow \mathbb{R}^{+}$along trajectories of (IVP) is

$$
\dot{V}_{\boldsymbol{u}}\left(\boldsymbol{x}_{0}\right):=\limsup _{t \rightarrow 0^{+}} \frac{1}{t}\left(V(\boldsymbol{x}(t))-V\left(\boldsymbol{x}_{0}\right)\right) .
$$

Definition 2.4: [15, Definition 11] (ISS Lyapunov Function) A continuous function $V: D(\subset \mathbb{X}) \rightarrow \mathbb{R}^{+}$is called an ISS Lyapunov function on $D$, if there exist $\psi_{1}, \psi_{2} \in \mathscr{K}_{\infty}$, $\alpha \in \mathscr{K}_{\infty}$, and $\sigma \in \mathscr{K}$ such that for all $\boldsymbol{x}_{0} \in \mathbb{X}, \boldsymbol{u}(t) \in$ $P C\left(\mathbb{R}^{+} ; \mathbb{U}\right)$,

$$
\psi_{1}(\|\boldsymbol{x}\|) \leq V(\boldsymbol{x}) \leq \psi_{2}(\|\boldsymbol{x}\|), \forall \boldsymbol{x} \in \mathbb{X},
$$

and

$$
\dot{V}_{\boldsymbol{u}}\left(\boldsymbol{x}_{0}\right) \leq-\alpha\left(\left\|\boldsymbol{x}_{0}\right\|\right)+\sigma\left(\sup _{t \geq 0}\|\boldsymbol{u}(t)\|_{\mathbb{U}}\right)
$$

\section{WELL-POSEDNESS OF RAILWAY TRACK MODEL}

Railway tracks rest on ballast which is known to exhibit nonlinear viscoelastic behavior. Considering the Kelvin-Voigt damping in the track beam, the semi-linear partial differential equation governing the motion of the track $w(\xi, t)$ on $\xi \in[0, \ell]$ is [8]

$$
\left\{\begin{array}{l}
\rho a \frac{\partial^{2} w}{\partial t^{2}}+\frac{\partial}{\partial \xi^{2}}\left(E I \frac{\partial^{2} w}{\partial \xi^{2}}+C_{d} \frac{\partial^{3} w}{\partial \xi^{2} \partial t}\right)+\mu \frac{\partial w}{\partial t}+k w \\
\quad+\alpha w^{3}=u(\xi, t), \\
w(\xi, 0)=w_{0}(\xi), \quad \frac{\partial w}{\partial t}(\xi, 0)=v_{0}(\xi), \\
w(0, t)=w(\ell, t)=0, \\
E I \frac{\partial^{2} w}{\partial \xi^{2}}(0, t)+C_{d} \frac{\partial^{3} w}{\partial \xi^{2} \partial t}(0, t)=0, \\
E I \frac{\partial^{2} w}{\partial \xi^{2}}(\ell, t)+C_{d} \frac{\partial^{3} w}{\partial \xi^{2} \partial t}(\ell, t)=0,
\end{array}\right.
$$

where the positive constants $E, I, \rho, a$, and $\ell$ are the modulus of elasticity, second moment of inertia, density of the beam, cross-sectional area, and length of the beam, respectively. The linear and nonlinear parts of the foundation elasticity correspond to the positive coefficients $k$ and $\alpha$, respectively. The constant $\mu>0$ is the damping coefficient of the foundation, and $C_{d} \geq 0$ is the coefficient of Kelvin-Voigt damping in the beam. The external force exerted on the railway track by moving trains, active dampers, or other external force, is denoted by $u(\xi, t)$. The model considered here differs from that in [8] by the inclusion of Kelvin-Voigt damping in the beam if $C_{d}$ has a non-zero value, although $C_{d}>0$ is not assumed in the analysis.

Let $v=\partial w / \partial t$. Define the state space $\mathbb{X}=H^{2}(0, \ell) \cap$ $H_{0}^{1}(0, \ell) \times L^{2}(0, \ell)$ with norm

$$
\|(w, v)\|^{2}=\int_{0}^{\ell} E I w_{\xi \xi}^{2}+k w^{2}+\rho a v^{2} d \xi,
$$

where the subscript $\cdot \xi$ denotes the derivative with respect to $\xi$. Define the closed self-adjoint positive operator

$$
\begin{aligned}
\mathcal{A}_{0} w:= & w_{\xi \xi \xi \xi}, \\
D\left(\mathcal{A}_{0}\right):= & \left\{w \in H^{4}(0, \ell) \mid w(0)=w(\ell)=0,\right. \\
& \left.w_{\xi \xi}(0)=w_{\xi \xi}(\ell)=0\right\},
\end{aligned}
$$

and also define

$$
\mathcal{A}_{K V}(w, v):=\left(v,-\frac{1}{\rho a} \mathcal{A}_{0}\left(E I w+C_{d} v\right)\right),
$$

with

$$
\begin{aligned}
D\left(\mathcal{A}_{K V}\right):= & \left\{(w, v) \in \mathbb{X} \mid v \in H^{2}(0, \ell) \cap H_{0}^{1}(0, \ell),\right. \\
& \left.E I w+C_{d} v \in D\left(\mathcal{A}_{0}\right)\right\} .
\end{aligned}
$$

Also, let $\boldsymbol{u} \in \mathbb{U}:=L^{2}(0, \ell)$, and define the linear operators $\mathcal{K}, \mathcal{A}, \mathcal{B}$, and the nonlinear operator $\mathcal{F}(\cdot)$ as

$$
\begin{aligned}
& \mathcal{K}(w, v):=\left(0,-\frac{1}{\rho a}(\mu v+k w)\right), \\
& \mathcal{A}:=\mathcal{A}_{K V}+\mathcal{K}, \text { with } D(\mathcal{A})=D\left(\mathcal{A}_{K V}\right), \\
& \mathcal{B} \boldsymbol{u}:=\left(0, \frac{1}{\rho a} \boldsymbol{u}\right), \\
& \mathcal{F}(w, v):=\left(0,-\frac{\alpha}{\rho a} w^{3}\right) .
\end{aligned}
$$


With these definitions and by setting the state $\boldsymbol{x}(t)=$ $(w(\cdot, t), v(\cdot, t))$, initial condition $\boldsymbol{x}_{0}=\left(w_{0}(\cdot), v_{0}(\cdot)\right)$, and the input $\boldsymbol{u}(t)=u(\cdot, t)$, the state space representation of the railway track IVP is

$$
\left\{\begin{array}{l}
\dot{\boldsymbol{x}}(t)=\mathcal{A} \boldsymbol{x}(t)+\mathcal{F}(\boldsymbol{x}(t))+\mathcal{B} \boldsymbol{u}(t), t>0, \\
\boldsymbol{x}(0)=\boldsymbol{x}_{0} \in \mathbb{X} .
\end{array}\right.
$$

Notice that the nonlinear term $w^{3}$ is in $L^{2}(0, \ell)$ since $H^{2}(0, \ell) \subset C([0, \ell])$. Thus, the nonlinear operator $\mathcal{F}(\cdot)$ is well-defined on $\mathbb{X}$. It is also locally Lipschitz continuous; see [26, Lem. 6.1] where it is shown to be continuously Fréchet differentiable on $\mathbb{X}$. Also, the bounded operator $\mathcal{B}$ maps an input $\boldsymbol{u} \in L^{2}(0, \ell)$ into the state space $\mathbb{X}$. This input space is used in many applications.

It is well known that $\mathcal{A}_{K V}$ generates a strongly continuous contraction semigroup on $\mathbb{X}$; see [27]. The operator $\mathcal{K}$ is a bounded linear operator on $\mathbb{X}$ and so the operator $\mathcal{A}$, with the same domain as $\mathcal{A}_{K V}$, generates a strongly continuous semigroup on $\mathbb{X}[25$, Cor. 3.2.2]. The assumption that $\mu>0$ implies that $\mathcal{A}$ generates an exponentially stable semigroup.

Theorem 3.1: [26, Theorem 3.1] Let $\mathcal{A}$ be the infinitesimal generator of a strongly continuous semigroup. If the nonlinear operator $\mathcal{F}(\cdot)$ is locally Lipschitz continuous on $\mathbb{X}$, then for every $\boldsymbol{x}_{0} \in \mathbb{X}$ and positive number $R$, there exist $T>0$ such that (IVP) admits a unique mild solution $\boldsymbol{x} \in C([0, T] ; \mathbb{X})$ for all $\boldsymbol{u} \in L^{p}(0, T ; \mathbb{U}),\|\boldsymbol{u}\|_{L^{p}(0, T ; \mathbb{U})} \leq R, 1<p<\infty$.

Thus, by Theorem 3.1, a unique local (in time) mild solution to railway IVP is ensured.

If the input admits further regularity, a mild solution is also a classical solution.

Theorem 3.2: [25, Theorem 6.1.5] Let $\mathcal{A}$ be the infinitesimal generator of a strongly continuous semigroup $\mathcal{T}(t)$ on $\mathbb{X}$. If $\boldsymbol{u} \in C^{1}([0, T] ; \mathbb{U})$ and the nonlinear operator $\mathcal{F}(\cdot)$ is continuously Fréchet differentiable on $\mathbb{X}$, then the mild solution of (IVP) with $\boldsymbol{x}_{0} \in D(\mathcal{A})$ is a classical solution.

\section{STABILITY OF CLASSICAL SOLUTIONS}

The existing literature does not predict the existence of a global solution to the railway track PDE model. To investigate the existence and stability of a global solution, a Lyapunov method [28] together with the multiplier method [23] is used. Let $c$ be a constant to be determined. Define, for any $\boldsymbol{x} \in \mathbb{X}$,

$$
\begin{aligned}
& V(\boldsymbol{x})= \\
& \quad \int_{0}^{\ell} E I\left(w_{\xi \xi}\right)^{2}+k w^{2}+\frac{\alpha}{2} w^{4}+\rho a v^{2}+2 c w v d \xi .
\end{aligned}
$$

Lemma 4.1: Let $c$ satisfy

$$
0<c<\sqrt{\rho k a} .
$$

Then, there exist positive numbers $c_{l}, c_{u}$, and $c_{e}$ such that for every $\boldsymbol{x} \in \mathbb{X}$,

$$
c_{l}\|\boldsymbol{x}\|^{2} \leq V(\boldsymbol{x}) \leq c_{u}\|\boldsymbol{x}\|^{2}+c_{h}\|\boldsymbol{x}\|^{4} .
$$

Proof: Young's inequality implies that for all $\epsilon_{1}>0$

$$
\left|\int_{0}^{\ell} 2 c w v d \xi\right| \leq c \int_{0}^{\ell} \epsilon_{1} w^{2}+\frac{1}{\epsilon_{1}} v^{2} d \xi .
$$

This inequality gives the following lower bound on $V$ :

$$
\begin{gathered}
V(\boldsymbol{x}) \geq \int_{0}^{\ell} E I\left(w_{\xi \xi}\right)^{2}+\left(k-c \epsilon_{1}\right) w^{2}+\frac{\alpha}{2} w^{4} \\
+\left(\rho a-\frac{c}{\epsilon_{1}}\right) v^{2} d \xi .
\end{gathered}
$$

Define

$$
c_{l}=\min \left\{1-\frac{c \epsilon_{1}}{k}, 1-\frac{c}{\rho a \epsilon_{1}}\right\} .
$$

The condition (20) on $c$ implies that there exists a number $\epsilon_{1}$ satisfying $c / \rho a<\epsilon_{1}<k / c$, which ensures that $c_{l}>0$. The inequality (23) can then be re-written as

$$
V(\boldsymbol{x}) \geq c_{l}\|\boldsymbol{x}\|^{2} \text {. }
$$

Furthermore, apply the inequality (22) to (19) and define $c_{u}=1+\max \left\{c \epsilon_{1} / k, c /\left(\epsilon_{1} \rho a\right)\right\}$ to obtain

$$
\begin{aligned}
V(\boldsymbol{x}) \leq & \int_{0}^{\ell} E I w_{\xi \xi}^{2}+\left(k+c \epsilon_{1}\right) w^{2}+\frac{\alpha}{2} w^{4} \\
& +\left(\rho a+\frac{c}{\epsilon_{1}}\right) v^{2} d \xi \\
\leq & c_{u}\|\boldsymbol{x}\|^{2}+\frac{\alpha}{2} \int_{0}^{\ell} w^{4} d \xi .
\end{aligned}
$$

Recall the continuous embedding $H^{2}(0, \ell) \hookrightarrow L^{4}(0, \ell)$. Letting $c_{e}$ be the embedding constant,

$$
\begin{aligned}
V(\boldsymbol{x}) & \leq c_{u}\|\boldsymbol{x}\|^{2}+\frac{\alpha}{2} \int_{0}^{\ell} w^{4} d \xi \\
& \leq c_{u}\|\boldsymbol{x}\|^{2}+\frac{\alpha c_{e}}{2}\left(\int_{0}^{\ell} w_{\xi \xi}^{2} d \xi\right)^{2} \\
& \leq c_{u}\|\boldsymbol{x}\|^{2}+\frac{\alpha c_{e}}{2(E I)^{2}}\|\boldsymbol{x}\|^{4} .
\end{aligned}
$$

Set $c_{h}=\alpha c_{e} / 2(E I)^{2}$ in the above inequality to complete the proof.

The derivative of the Lyapunov function along trajectories of the railway track IVP exists for every $\boldsymbol{x}_{0} \in D(\mathcal{A})$ and continuously differentiable input. In the next lemma, if $C_{d}=$ 0 , set $1 / C_{d}=\infty$.

Lemma 4.2: Let $[0, T]$ be the interval of existence of the classical solution $\boldsymbol{x}(t)$ to the railway track IVP with $\boldsymbol{x}_{0} \in$ $D(\mathcal{A})$ and $\boldsymbol{u} \in C^{1}([0, T] ; \mathbb{U})$. Then, the Lyapunov function $V(\boldsymbol{x}(t))$ is differentiable with respect to time. Moreover, let $c$ satisfy

$$
0<c<\min \left\{\frac{4 \rho a E I}{C_{d}}, \frac{4 \rho a k \mu}{\mu^{2}+4 \rho a k}, \frac{4 \rho a k \mu}{1+4 \rho a k}\right\} .
$$

Then, there are positive constants $\epsilon_{3}$ and $\omega$ such that the derivative $\dot{V}(\boldsymbol{x}(t))$ satisfies for all $t \in[0, T]$

$$
\dot{V}(\boldsymbol{x}(t)) \leq \epsilon_{3}\|\boldsymbol{u}(t)\|_{\mathbb{U}}^{2}-\omega V(\boldsymbol{x}(t))
$$

Proof: Since $\boldsymbol{x}_{0} \in D(\mathcal{A})$ and $\boldsymbol{u} \in C^{1}([0, T] ; \mathbb{U})$, the state $\boldsymbol{x}(t)$ is differentiable on $[0, T]$. The derivative of the Lyapunov function along trajectories of the railway track IVP is

$$
\begin{gathered}
\dot{V}(\boldsymbol{x}(t))=2 \int_{0}^{\ell} E I w_{\xi \xi} \dot{w}_{\xi \xi}+k w \dot{w}+\alpha w^{3} \dot{w}+\rho a \dot{v} v \\
+c \dot{w} v+c w \dot{v} d \xi .
\end{gathered}
$$


Substituting the time derivatives from the railway track model (10) leads to

$$
\begin{aligned}
& \dot{V}(\boldsymbol{x}(t))=2 \int_{0}^{\ell} E I w_{\xi \xi} v_{\xi \xi}+k w v+\alpha w^{3} v \\
& \quad-v\left(\left(E I w+C_{d} v\right)_{\xi \xi \xi \xi}+k w+\mu v+\alpha w^{3}+u(\xi, t)\right) \\
& \quad+c v^{2}-\frac{c}{\rho a} w\left(\left(E I w+C_{d} v\right)_{\xi \xi \xi \xi}+k w+\mu v\right. \\
& \left.\quad+\alpha w^{3}+u(\xi, t)\right) d \xi
\end{aligned}
$$

Performing repeated integration by parts and using the boundary conditions lead to

$$
\begin{aligned}
& \dot{V}(\boldsymbol{x}(t))=-2\left[\left(v+\frac{c}{\rho a} w\right)\left(E I w+C_{d} v\right)_{\xi \xi \xi}\right. \\
& \left.-\left(v_{\xi}+\frac{c}{\rho a} w_{\xi}\right)\left(E I w+C_{d} v\right)_{\xi \xi}\right]_{0}^{\ell} \\
& -2 \int_{0}^{\ell} \frac{E I c}{\rho a}\left(w_{\xi \xi}\right)^{2}+\frac{k c}{\rho a} w^{2}+\frac{\alpha c}{\rho a} w^{4}+(\mu-c) v^{2} \\
& +C_{d} v_{\xi \xi}^{2} d \xi-2 \int_{0}^{\ell} \frac{\mu c}{\rho a} w v+\frac{C_{d} c}{\rho a} w_{\xi \xi} v_{\xi \xi} d \xi \\
& -2 \int_{0}^{\ell} u(\xi, t)\left(v+\frac{c}{\rho a} w\right) d \xi .
\end{aligned}
$$

Young's inequalities (such as inequality (22)) are used to bound the product terms. Letting $\epsilon_{1}, \epsilon_{2}$ and $\epsilon_{3}$ be positive constants,

$$
\begin{aligned}
\dot{V}(\boldsymbol{x}(t)) \leq \epsilon_{3}\|\boldsymbol{u}(t)\|_{\mathbb{U}}^{2} & -\frac{2}{\rho a} \int_{0}^{\ell}\left(E I-\frac{C_{d} \epsilon_{2}}{2}\right) c\left(w_{\xi \xi}\right)^{2} \\
& +\left(k-\frac{\mu \epsilon_{1}}{2}-\frac{c}{2 \rho a \epsilon_{3}}\right) c w^{2}+\alpha c w^{4} \\
& +\left(\rho a \mu-\rho a c-\frac{\mu c}{2 \epsilon_{1}}-\frac{\rho a \epsilon_{3}}{2}\right) v^{2} \\
& +\left(\rho a-\frac{c}{2 \epsilon_{2}}\right) C_{d}\left(v_{\xi \xi}\right)^{2} d \xi
\end{aligned}
$$

Define the constant

$$
\begin{aligned}
\omega_{0}=\frac{2 c}{\rho a} \min \{ & 1-\frac{C_{d} \epsilon_{2}}{2 E I}, 1-\frac{\mu \epsilon_{1}}{2 k}-\frac{c}{2 \rho a \epsilon_{3} k}, \\
& \left.\frac{\mu}{c}-1-\frac{\mu}{2 \epsilon_{1} \rho a}-\frac{\epsilon_{3}}{2 c}, \frac{\rho a}{c}-\frac{1}{2 \epsilon_{2}}\right\} .
\end{aligned}
$$

The constant $\omega_{0}$ needs to be positive. Thus, the constants $\epsilon_{1}$, $\epsilon_{2}$, and $\epsilon_{3}$ are required to satisfy

$$
\begin{aligned}
0 & <\epsilon_{1}<\frac{2 k}{\mu}-\frac{c}{\rho a \epsilon_{3} \mu}, \\
\frac{c}{2 \rho a} & <\epsilon_{2}<\frac{2 E I}{C_{d}}, \\
0 & <\epsilon_{3}<2 \mu-2 c-\frac{\mu c}{\epsilon_{1} \rho a} .
\end{aligned}
$$

There is a positive number $\epsilon_{2}$ satisfying (36) if

$$
0<c<\frac{4 \rho a E I}{C_{d}}
$$

Also, inequalities (35) and (37) have a solution for $\epsilon_{1}$ and $\epsilon_{3}$ if

$$
0<c<\min \left\{\frac{4 \rho a k \mu}{\mu^{2}+4 \rho a k}, \frac{4 \rho a k \mu}{1+4 \rho a k}\right\} .
$$

Inequality (33) can then be re-written as

$$
\begin{aligned}
& \dot{V}(\boldsymbol{x}(t)) \leq \epsilon_{3}\|\boldsymbol{u}(t)\|_{\mathbb{U}}^{2} \\
& -\omega_{0} \int_{0}^{\ell} E I\left(w_{\xi \xi}\right)^{2}+k w^{2}+\alpha w^{4}+\rho a v^{2}+C_{d}\left(v_{\xi \xi}\right)^{2} d \xi .
\end{aligned}
$$

Using Young's inequality, an upper bound on the Lyapunov function is

$$
V(\boldsymbol{x}(t)) \leq r \int_{0}^{\ell} E I\left(w_{\xi \xi}\right)^{2}+k w^{2}+\alpha w^{4}+\rho a v^{2} d \xi,
$$

where

$$
r=1+\max \left\{\frac{c \epsilon_{1}}{k}, \frac{c}{\rho a \epsilon_{2}}\right\} .
$$

Use this upper bound in (40)

$$
\dot{V}(\boldsymbol{x}(t)) \leq \epsilon_{3}\|\boldsymbol{u}(t)\|_{\mathbb{U}}^{2}-\frac{\omega_{0}}{r} V(\boldsymbol{x}(t)) .
$$

Set $\omega=\omega_{0} / r$ to complete the proof.

The next theorem uses the Lyapunov function to show that a unique classical solution exists on arbitrary intervals of time for a large class of inputs. It also ensures exponential stability of the solution for some inputs.

Theorem 4.3: Let $c_{l}, c_{u}, c_{h}, \omega$, and $\epsilon_{3}$ be the same constants as in Lemma 4.1 and Lemma 4.2. If $\boldsymbol{x}_{0} \in D(\mathcal{A})$ and $\boldsymbol{u} \in C^{1}\left(\mathbb{R}^{+} ; \mathbb{U}\right)$, then the unique classical solution $\boldsymbol{x}(t)$ to the railway track IVP exists for all $t \geq 0$ and satisfies

$$
\begin{aligned}
\|\boldsymbol{x}(t)\|^{2} \leq e^{-\omega t} & \left(\frac{c_{u}}{c_{l}}\left\|\boldsymbol{x}_{0}\right\|^{2}+\frac{c_{h}}{c_{l}}\left\|\boldsymbol{x}_{0}\right\|^{4}\right) \\
& +\frac{\epsilon_{3}}{\omega}\left(1-e^{-\omega t}\right) \max _{s \in[0, t]}\|\boldsymbol{u}(s)\|_{\mathbb{U}}^{2} .
\end{aligned}
$$

Moreover, if there are positive constants $u_{0}$ and $\delta$ so that $\|\boldsymbol{u}(t)\|_{\mathbb{U}} \leq u_{0} e^{-\delta t}$, then $\|\boldsymbol{x}(t)\|$ exponentially decays to zero.

Proof: For every $\bar{T}>0$, consider the input $\boldsymbol{u}$ over the bounded interval $[0, \bar{T}]$ and define $R:=\|\boldsymbol{u}\|_{L^{2}(0, \bar{T} ; \mathbb{U})}$. According to Theorem 3.1 and 3.2 , for every $x_{0} \in D(\mathcal{A})$ and $\boldsymbol{u} \in C^{1}([0, \bar{T}] ; \mathbb{U})$, with $\|\boldsymbol{u}\|_{L^{2}(0, \bar{T} ; \mathbb{U})} \leq R$, there is an interval $[0, T], T=T\left(\boldsymbol{x}_{0}, R\right) \leq \bar{T}$, over which a classical solution to the railway track model (10) exists.

Now use Lemma 4.2, and apply Grönwall's lemma [29, Thm. 1.4.1] to inequality (29) to obtain

$$
V(\boldsymbol{x}(t)) \leq e^{-\omega t} V\left(\boldsymbol{x}_{0}\right)+\epsilon_{3} \int_{0}^{t} e^{-\omega(t-s)}\|\boldsymbol{u}(s)\|_{\mathbb{U}}^{2} d s,
$$

for all $t \in[0, T]$. This yields

$$
V(\boldsymbol{x}(t)) \leq e^{-\omega t} V\left(\boldsymbol{x}_{0}\right)+\frac{\epsilon_{3}}{\omega}\left(1-e^{-\omega t}\right) \max _{s \in[0, t]}\|\boldsymbol{u}(s)\|_{\mathbb{U}}^{2} .
$$

From Lemma 4.1, it follows that for all $t \in[0, T]$,

$$
\begin{aligned}
\|\boldsymbol{x}(t)\|^{2} \leq e^{-\omega t} & \left(\frac{c_{u}}{c_{l}}\left\|\boldsymbol{x}_{0}\right\|^{2}+\frac{c_{h}}{c_{l}}\left\|\boldsymbol{x}_{0}\right\|^{4}\right) \\
& +\frac{\epsilon_{3}}{\omega}\left(1-e^{-\omega t}\right) \max _{t \in[0, \bar{T}]}\|\boldsymbol{u}(t)\|_{\mathbb{U}}^{2} .
\end{aligned}
$$

This classical solution is of course also a mild solution on $[0, T]$. Note that $\|\boldsymbol{x}(t)\| \leq M$ where $M$ is independent of $T$. Using [25, Thm. 6.1.4] the solution $\boldsymbol{x}(t)$ can be extended to $[0, \bar{T}]$. Since $\bar{T}$ was arbitrary, the mild solution exists for all 
$t>0$. Since $\boldsymbol{x}_{0} \in D(\mathcal{A})$ and $\boldsymbol{u} \in C^{1}\left(\mathbb{R}^{+} ; \mathbb{U}\right)$, Theorem 3.2 then implies that this mild solution is also a classical solution.

Furthermore, if there are positive constants $u_{0}$ and $\delta$ so that $\|\boldsymbol{u}(t)\|_{\mathbb{U}} \leq u_{0} e^{-\delta t}$, inequality (45) yields

$$
V(\boldsymbol{x}(t)) \leq e^{-\omega t} V\left(\boldsymbol{x}_{0}\right)+\epsilon_{3} u_{0}^{2} \begin{cases}\frac{e^{-2 \delta t}-e^{-\omega t}}{\omega-2 \delta} & \omega \neq 2 \delta \\ e^{-\omega t} & \omega=2 \delta\end{cases}
$$

This shows that the Lyapunov function exponentially decays to zero. Since the Lyapunov function $V(\boldsymbol{x})$ bounds the norm of the state by Lemma 4.1, the state will also exponentially decay to zero.

For inputs with $\sup _{t \geq 0}\|\boldsymbol{u}(t)\|_{\mathbb{U}}<\infty$, Lemma 4.1 and Lemma 4.2 result in

$$
\dot{V}(\boldsymbol{x}(t)) \leq-\omega c_{l}\|\boldsymbol{x}(t)\|^{2}+\epsilon_{3} \sup _{t \geq 0}\|\boldsymbol{u}(t)\|_{\mathbb{U}}^{2} .
$$

From Definition 2.4, this inequality shows that the Lyapunov function is an ISS Lyapunov function on $D=D(\mathcal{A})$.

\section{STABILITY OF MILD SOLUTIONS}

If the initial condition is not in $D(\mathcal{A})$ or the input is not continuously differentiable, there may be a unique mild solution to (IVP) even though a classical solution may not exist. In this case, the Lyapunov function from Theorem 4.3 may not be differentiable. Thus, exponential decay cannot be shown through manipulating the derivative of Lyapunov function. However, the proof of Theorem 4.3 can be modified to yield a result that ensures the existence and stability of global mild solutions for initial conditions in $\mathbb{X}$ and inputs in $L_{l o c}^{2}(0, \infty ; \mathbb{U})$.

Theorem 5.1: Let $c_{l}, c_{u}, c_{h}, \omega$, and $\epsilon_{3}$ be the same constants as in Lemma 4.1 and Lemma 4.2. If $\boldsymbol{x}_{0} \in \mathbb{X}$ and $\boldsymbol{u} \in L_{l o c}^{2}(0, \infty ; \mathbb{U})$, then the unique mild solution, $\boldsymbol{x}(t)$, to the railway track IVP exists globally. For every $t>0$, the mild solution satisfies

$$
\begin{aligned}
\|\boldsymbol{x}\|_{C(0, t ; \mathbb{X})}^{2}+\omega\|\boldsymbol{x}\|_{L^{2}(0, t ; \mathbb{X})}^{2} \leq & \frac{c_{u}}{c_{l}}\left\|\boldsymbol{x}_{0}\right\|^{2}+\frac{c_{h}}{c_{l}}\left\|\boldsymbol{x}_{0}\right\|^{4} \\
& +\frac{\epsilon_{3}}{c_{l}}\|\boldsymbol{u}\|_{L^{2}(0, t ; \mathbb{U})}^{2} .
\end{aligned}
$$

Proof: For every $\bar{T}>0$, consider the input $\boldsymbol{u}$ over the bounded interval $[0, \bar{T}]$ and define $R:=\|\boldsymbol{u}\|_{L^{2}(0, \bar{T} ; \mathbb{U})}$. According to Theorem 3.1, for every $x_{0} \in \mathbb{X}$ and $R$, there is an interval $[0, T], T \leq \bar{T}$, over which a unique mild solution $\boldsymbol{x}(t)$ to the railway track IVP exists. Pick a sequence $\boldsymbol{u}_{n} \in C^{1}([0, T] ; \mathbb{U})$, with $\left\|\boldsymbol{u}_{n}\right\|_{L^{2}(0, T ; \mathbb{U})} \leq R$, for all $n \in \mathbb{N}$, that converges to $\boldsymbol{u}$ in $L^{2}(0, T ; \mathbb{U})$. Also, pick a sequence of initial conditions $x_{0}^{n} \in D(\mathcal{A})$ that converges to $\boldsymbol{x}_{0}$ in $\mathbb{X}$. Such sequences always exist since $C^{1}([0, T], \mathbb{U})$ is dense in $L^{2}([0, T] ; \mathbb{U})$, and $D(\mathcal{A})$ is densely embedded in $\mathbb{X}$. Corresponding to each initial condition $x_{0}^{n}$ and input $\boldsymbol{u}_{n}(t)$ is a unique classical solution $\boldsymbol{x}_{n}(t), t \in[0, T]$, ensured by Theorem 3.2. This sequence of solutions also satisfies the equation (5) of mild solutions. The mild solution (5) is continuous with respect to $\boldsymbol{x}_{0} \in \mathbb{X}$ and $\boldsymbol{u} \in L^{2}(0, T ; \mathbb{U})$. See [25, Thm. 6.1.2] for Lipschitz continuity with respect to initial conditions, and [26, Proposition 5.2] for Lipschitz continuity with respect to inputs. It follows that

$$
\boldsymbol{x}_{n} \rightarrow \boldsymbol{x} \text { in } C([0, T] ; \mathbb{X})
$$

as $\boldsymbol{x}_{0}^{n} \rightarrow \boldsymbol{x}_{0}$ in $\mathbb{X}$ and $\boldsymbol{u}_{n} \rightarrow \boldsymbol{u}$ in $L^{2}(0, T ; \mathbb{U})$.

The Lyapunov function is differentiable for every pair $\left(\boldsymbol{x}_{n}(t), \boldsymbol{u}_{n}(t)\right)$, and from Lemma 4.2, its derivative satisfies

$$
\dot{V}\left(\boldsymbol{x}_{n}(t)\right) \leq \epsilon_{3}\left\|\boldsymbol{u}_{n}(t)\right\|_{\mathbb{U}}^{2}-\omega V\left(\boldsymbol{x}_{n}(t)\right),
$$

for all $t \in[0, T]$. Taking the integral yields

$$
\begin{aligned}
V\left(\boldsymbol{x}_{n}(t)\right) \leq & V\left(\boldsymbol{x}_{0}^{n}\right)+\epsilon_{3} \int_{0}^{t}\left\|\boldsymbol{u}_{n}(s)\right\|_{\mathbb{U}}^{2} d s \\
& -\omega \int_{0}^{t} V\left(\boldsymbol{x}_{n}(s)\right) d s .
\end{aligned}
$$

From Lemma 4.1, the Lyapunov function satisfies

$$
\begin{aligned}
\left|V\left(\boldsymbol{x}_{2}\right)-V\left(\boldsymbol{x}_{1}\right)\right| \leq c_{u} \mid & \left\|\boldsymbol{x}_{2}\right\|^{2}-\left\|\boldsymbol{x}_{1}\right\|^{2} \mid \\
& +c_{h}\left|\left\|\boldsymbol{x}_{2}\right\|^{4}-\left\|\boldsymbol{x}_{1}\right\|^{4}\right|,
\end{aligned}
$$

for all $\boldsymbol{x}_{1}$ and $\boldsymbol{x}_{2}$ in $\mathbb{X}$. After some manipulation, it follows that

$$
\begin{aligned}
& \left|V\left(\boldsymbol{x}_{2}\right)-V\left(\boldsymbol{x}_{1}\right)\right| \leq \\
& \quad\left(c_{u}+c_{h}\left(\left\|\boldsymbol{x}_{2}\right\|^{2}+\left\|\boldsymbol{x}_{1}\right\|^{2}\right)\right)\left(\left\|\boldsymbol{x}_{2}\right\|+\left\|\boldsymbol{x}_{1}\right\|\right)\left\|\boldsymbol{x}_{2}-\boldsymbol{x}_{1}\right\| .
\end{aligned}
$$

Thus, for every $s \in[0, T]$, the sequence $V\left(\boldsymbol{x}_{n}(s)\right)$ converges to $V(\boldsymbol{x}(s))$. The convergence is also uniform; that is, define

$$
r=\sup _{n}\left\|\boldsymbol{x}_{n}\right\|_{C([0, T] ; \mathbb{X})},
$$

find that

$$
\begin{aligned}
& \left|V\left(\boldsymbol{x}_{n}(s)\right)-V(\boldsymbol{x}(s))\right| \leq \max _{s \in[0, T]}\left|V\left(\boldsymbol{x}_{n}(s)\right)-V(\boldsymbol{x}(s))\right| \\
& \leq\left(2 c_{u} r+4 c_{h} r^{3}\right)\left\|\boldsymbol{x}_{n}-\boldsymbol{x}\right\|_{C([0, T] ; \mathbb{X})} .
\end{aligned}
$$

Thus, by the uniform convergence theorem, the integral in (52) converges. These together with (50) imply that

$$
V(\boldsymbol{x}(t))+\omega \int_{0}^{t} V(\boldsymbol{x}(s)) d s \leq V\left(\boldsymbol{x}_{0}\right)+\epsilon_{3} \int_{0}^{t}\|\boldsymbol{u}(s)\|_{\mathbb{U}}^{2} d s
$$

for all $t \in[0, T]$. Apply Lemma 4.1 to this inequality and take the maximum of both side over $[0, t]$ to obtain

$$
\begin{aligned}
c_{l}\|\boldsymbol{x}\|_{C(0, t ; \mathbb{X})}^{2}+\omega c_{l}\|\boldsymbol{x}\|_{L^{2}(0, t ; \mathbb{X})}^{2} & \leq c_{u}\left\|\boldsymbol{x}_{0}\right\|^{2}+c_{h}\left\|\boldsymbol{x}_{0}\right\|^{4} \\
& +\epsilon_{3}\|\boldsymbol{u}\|_{L^{2}(0, t ; \mathbb{U})}^{2}
\end{aligned}
$$

This inequality shows that the mild solution can be extended to the interval $[0, \bar{T}][25$, Thm. 6.1.4]. Since $\bar{T}$ was arbitrary, the mild solution exists globally.

For inputs in $P C\left(\mathbb{R}^{+} ; \mathbb{U}\right)$, a similar density argument can be applied to prove the ISS of railway track IVP.

Corollary 5.2: The railway track IVP is input-to-state stable (ISS) in the sense of Definition 2.3.

Proof: For every $T>0, P C([0, T] ; \mathbb{U}) \subset L_{l o c}^{2}(0, \infty ; \mathbb{U})$; thus, Theorem 5.1 ensures that a unique mild solution $\boldsymbol{x}(t)$ exists for all inputs $\boldsymbol{u}$ in $P C([0, T] ; \mathbb{U})$. Consider a sequence of initial conditions $\boldsymbol{x}_{0}^{n} \in D(\mathcal{A})$ converging to $\boldsymbol{x}_{0}$ in $\mathbb{X}$, and also 
a sequence of inputs $\boldsymbol{u}_{n} \in C^{1}([0, T] ; \mathbb{U})$ converging uniformly to $\boldsymbol{u} \in P C([0, T] ; \mathbb{U})$. Let $\boldsymbol{x}_{n}(t)$ be the classical solution to railway track IVP with initial condition $x_{0}^{n}$ and input $\boldsymbol{u}_{n}(t)$. This solution also satisfies (5) which ensures that

$$
\begin{gathered}
\boldsymbol{x}_{n} \rightarrow \boldsymbol{x} \text { in } C([0, T] ; \mathbb{X}) \\
\text { as } \boldsymbol{x}_{0}^{n} \rightarrow \boldsymbol{x}_{0} \text { in } \mathbb{X} \text { and } \boldsymbol{u}_{n}(t) \rightarrow \boldsymbol{u}(t) \text { uniformly. }
\end{gathered}
$$

See [25, Thm. 6.1.2] for Lipschitz continuity with respect to initial conditions, and [26, Proposition 5.2] for Lipschitz continuity with respect to inputs. Use Theorem 4.3 to obtain

$$
\begin{aligned}
\left\|\boldsymbol{x}_{n}(t)\right\|^{2} \leq e^{-\omega t} & \left(\frac{c_{u}}{c_{l}}\left\|\boldsymbol{x}_{0}^{n}\right\|^{2}+\frac{c_{h}}{c_{l}}\left\|\boldsymbol{x}_{0}^{n}\right\|^{4}\right) \\
& +\frac{\epsilon_{3}}{\omega} \max _{t \in[0, T]}\left\|\boldsymbol{u}_{n}(t)\right\|_{\mathbb{U}}^{2} .
\end{aligned}
$$

This inequality continuously depends on the norm of initial conditions and inputs. Taking the limit yields a similar inequality for $\boldsymbol{x}(t)$ with $\boldsymbol{x}_{0}$ and $\boldsymbol{u}(t)$ replaced. Knowing that $\sup _{t \geq 0}\|\boldsymbol{u}(t)\|_{\mathbb{U}}<\infty$, the ISS property in Definition 2.3 follows immediately.

\section{COnClusions And Future Research}

The stability and well-posedness of a nonlinear railway track model was established in this paper. Using a suitable Lyapunov function, it was proved that the model admits a global (in time) classical solution for a continuously differentiable input. The solution is also exponentially stable. For less regular inputs, belonging only to $L_{l o c}^{2}(0, \infty ; \mathbb{U})$ or $P C\left(\mathbb{R}^{+} ; \mathbb{U}\right)$, existence and stability of a mild solution as well as input-to-state stability (ISS) of the model were established. Current research is concerned with extending the results of this paper to more general nonlinear structural models.

\section{REFERENCES}

[1] T. Fu Ma, "Boundary stabilization for a non-linear beam on elastic bearings," Mathematical Methods in the Applied Sciences, vol. 24, no. 8, pp. 583-594, 2001.

[2] E. Fašangová and J. Prüss, "Asymptotic behaviour of a semilinear viscoelastic beam model," Archiv der Mathematik, vol. 77, no. 6, pp. 488-497, 2001.

[3] H. Takeda and S. Yoshikawa, "On the initial value problem of the semilinear beam equation with weak damping II: Asymptotic profiles," Journal of Differential Equations, vol. 253, no. 11, pp. 3061-3080, 2012.

[4] M. S. Edalatzadeh, R. Vatankhah, and A. Alasty, "Suppression of dynamic pull-in instability in electrostatically actuated strain gradient beams," in 2014 Second RSI/ISM International Conference on Robotics and Mechatronics (ICRoM), oct 2014, pp. 155-160.

[5] M. S. Edalatzadeh and A. Alasty, "Boundary exponential stabilization of non-classical micro/nano beams subjected to nonlinear distributed forces," Applied Mathematical Modelling, vol. 40, no. 3, pp. 2223-2241, 2016.

[6] W. Liu, K. Chen, and J. Yu, "Asymptotic stability for a non-autonomous full von Kármán beam with thermo-viscoelastic damping," Applicable Analysis, vol. 97, no. 3, pp. 400-414, 2018.

[7] M. Grasselli, G. Schimperna, and S. Zelik, "On the 2D Cahn-Hilliard equation with inertial term," Communications in Partial Differential Equations, vol. 34, no. 2, pp. 137-170, 2009.

[8] M. Ansari, E. Esmailzadeh, and D. Younesian, "Frequency analysis of finite beams on nonlinear Kelvin-Voight foundation under moving loads," Journal of Sound and Vibration, vol. 330, no. 7, pp. 1455-1471, 2011.

[9] T. Dahlberg, "Dynamic interaction between train and nonlinear railway track model," in Proc. Fifth Euro. Conf. Struct. Dyn., Munich, Germany, 2002, pp. 1155-1160.
[10] H. Ding, K.-L. Shi, L.-Q. Chen, and S.-P. Yang, "Dynamic response of an infinite Timoshenko beam on a nonlinear viscoelastic foundation to a moving load," Nonlinear Dynamics, vol. 73, no. 1-2, pp. 285-298, 2013.

[11] H. Ding, L.-Q. Chen, and S.-P. Yang, "Convergence of Galerkin truncation for dynamic response of finite beams on nonlinear foundations under a moving load," Journal of Sound and Vibration, vol. 331, no. 10, pp. 2426-2442, 2012.

[12] G. Kouroussis, C. Caucheteur, D. Kinet, G. Alexandrou, O. Verlinden, and V. Moeyaert, "Review of trackside monitoring solutions: from strain gages to optical fibre sensors," Sensors, vol. 15, no. 8, pp. $20115-20139$, 2015.

[13] S. Zhu, J. Yang, H. Yan, L. Zhang, and C. Cai, "Low-frequency vibration control of floating slab tracks using dynamic vibration absorbers," Vehicle System Dynamics, vol. 53, no. 9, pp. 1296-1314, 2015.

[14] A. Mironchenko, "Local input-to-state stability: Characterizations and counterexamples," Systems and Control Letters, vol. 87, pp. 23-28, 2016.

[15] A. Mironchenko and F. Wirth, "Characterizations of Input-to-State Stability for Infinite-Dimensional Systems," IEEE Transactions on Automatic Control, vol. 63, no. 6, pp. 1602-1617, 2018.

[16] B. Jayawardhana, H. Logemann, and E. P. Ryan, "Infinite-dimensional feedback systems: the circle criterion and input-to-state stability," Communications in Information and Systems, vol. 8, no. 4, pp. 413-444, 2008.

[17] F. Mazenc and C. Prieur, "Strict Lyapunov functions for semilinear parabolic partial differential equations," Mathematical Control and Related Fields, vol. 1, no. 2, pp. 231-250, 2011.

[18] A. Mironchenko and H. Ito, "Characterizations of integral input-tostate stability for bilinear systems in infinite dimensions." Mathematical Control and Related Fields, vol. 6, no. 3, pp. 447-466, 2016.

[19] B. Jacob, R. Nabiullin, J. R. Partington, and F. L. Schwenninger, "Infinite-dimensional input-to-state stability and Orlicz spaces," SIAM Journal on Control and Optimization, vol. 56, no. 2, pp. 868-889, 2018.

[20] I. Karafyllis and M. Krstic, "ISS with respect to boundary disturbances for 1-D parabolic PDEs," IEEE Transactions on Automatic Control, vol. 61, no. 12, pp. 3712-3724, 2016.

[21] Y. Orlov, "On General Properties of Eigenvalues and Eigenfunctions of a Sturm-Liouville Operator: Comments on ISS With Respect to Boundary Disturbances for 1-D Parabolic PDEs," IEEE Transactions on Automatic Control, vol. 62, no. 11, pp. 5970-5973, 2017.

[22] A. Pisano and Y. Orlov, "On the ISS properties of a class of parabolic DPS' with discontinuous control using sampled-in-space sensing and actuation," Automatica, vol. 81, pp. 447-454, 2017.

[23] V. Komornik, Exact controllability and stabilization: the multiplier method. Masson, 1994, vol. 36.

[24] A. Mironchenko, "Input-to-state stability of infinite-dimensional control systems," Ph.D. dissertation, University of Bremen, 2012.

[25] A. Pazy, Semigroups of linear operators and applications to partial differential equations. Springer Science \& Business Media, 2012, vol. 44.

[26] M. S. Edalatzadeh and K. A. Morris, "Optimal Actuator Location for Semi-linear Systems," arXiv preprint arxiv:1802.05807, 2018.

[27] S. P. Chen and R. Triggiani, "Proof of extensions of two conjectures on structural damping for elastic systems," Pacific Journal of Mathematics, vol. 136, no. 1, pp. 15-55, 1989.

[28] M. S. de Queiroz, D. M. Dawson, S. P. Nagarkatti, and F. Zhang, Lyapunov-based control of mechanical systems, ser. Control Engineering. Birkhäuser Boston, Inc., Boston, MA, 2000.

[29] A. Zettl, Sturm-Liouville Theory, ser. Mathematical Surveys and Monographs. American Mathematical Society, 2005. 